Professor Weale must be fully aware that no writer is ever consulted about publication price. Weale admits his opinion may be 'a little on the churlish side.' Agreed. Of course, I would have phrased it differently.

1350 West Seventh Street,

DAVID D. MICHAELS

San Pedro,

California 90732

USA.

\section{Contrast sensitivity testing}

SIR, A recent article ${ }^{1}$ reports an important methodological improvement for contrast sensitivity testing with printed gratings derived from the Arden plates. $^{2}$ Vaegan and Halliday ${ }^{1}$ used a 4-alternative forced-choice procedure to reduce the influence of response bias, and presented results obtained in glaucoma. However, in discussing these results they inaccurately characterised our previous findings on contrast sensitivity defects in glaucoma.$^{3-5}$ The inaccuracies have implications which concern not only testing methodology but also the pathophysiology of vision changes in glaucoma.

Vaegan and Halliday' found that 'glaucoma patients ... have a contrast sensitivity deficit of about $6 \mathrm{db}$ in relation to aged matched normal persons at all spatial frequencies when tested with a conventional oscilloscope display of static gratings' (p. 481). ${ }^{1}$ They erroneously conclude (p. 481) ${ }^{1}$ that their results 'disagree with the findings of Atkin et al. ${ }^{3}$,' and speculate (p. 490) that 'It is possible that the sample was too small or unrepresentative in many studies which do not find significant differences' (citing Atkin $e t$ al. ${ }^{35}$ ). There actually is no disagreement. Our results with static patterns (unpublished) were similar to Veagan and Halliday's. ${ }^{1}$ The static stimuli, which were similar to those used in conventional contrast sensitivity methods, ${ }^{12}$ had in fact yielded significant differences between group means but had not shown a high enough ability to discriminate individual patients from normals to offer promise of a clinically useful test. Therefore in our original abstract we had said that 'sensitivity to the (non-flickering) grating . . . did not distinguish between patients and controls', ${ }^{3}$ meaning that the groups showed such a large amount of overlap that the static-target method provided little useful information about individual patients. Consequently our subsequent papers $^{45}$ reported only the results with flickering targets.

With targets flickering at $8 \mathrm{~Hz}$ (a method not used by Vaegan and Halliday'), our studies revealed dynamic components of early glaucomatous vision changes ${ }^{45}$ which appeared to be more clinically useful than the static response data. Veagan and Halliday ${ }^{1}$ seemed in fact to recognise this possibility, for they suggested (on p. 490) that "Atkin et al. ${ }^{45}$ may .. . have had greater success with phase-alternating stimuli because responses to them are more reliable...' Exactly so! With dynamic testing there had been nearly perfect discrimination between glaucoma patients and age-matched control subjects. ${ }^{4}$ We had therefore concluded that the dynamic method might be useful to characterise individuals, not just groups.

It is important to call attention to this methodological difference-the use of dynamic rather than static contrast sensitivity measures. Since our original publications ${ }^{3-5}$ there have been several studies corroborating our emphasis on the importance of temporal modulation for discerning early effects in glaucoma, all suggesting that an abnormality in glaucomatous visual responses is apparent with particular clarity at moderate frequencies, e.g. $8 \mathrm{~Hz},{ }^{6-11}$ as well as at higher frequencies. ${ }^{6}$

Disparities in test sensitivity between dynamic and static methods may have pathophysiological implications. The higher sensitivity found for tests using flickering targets tends to support 2 of the several pathophysiological hypotheses that have been proposed to account for vision changes in glaucoma. (1) The first hypothesis was that there might be an early differential vulnerability of one component of retinal organisation (perhaps a subclass of retinal ganglion cells) which is especially sensitive when stimulated with fast temporal frequencies and less sensitive when stimulated with static gratings. ${ }^{28}$ (2) A subsequent suggestion was that vision changes might result in part from reduced synaptic efficacy within the CNS, due to depression of the rapid component of axoplasmic flow in optic nerve axons. ${ }^{12}$. The resulting neurotransmitter depletion could lead to a generalised reduction of visual sensitivity ${ }^{12}$ that would be exacerbated by temporal modulation, therefore showing greater effects with dynamic than with static testing.

We offer the foregoing comments to set the record straight about statements in the Vaegan and Halliday paper ${ }^{1}$ which could cause confusion.

Departments of Ophthalmology

ADAM ATKIN, and Neurology,

The Mount Sinai School of Medicine, New York, NY 10029, USA.

IVAN BODIS-WOLLNER, MURRAY WOLKSTEIN, STEVEN PODOS.

\section{References}

1 Vaegan, Halliday BL. A forced-choice test improves clinical contrast sensitivity testing. Br J Ophthalmol 1982; 66: 477-91.

2 Arden GB. The importance of measuring contrast sensitivity in cases of visual disturbance. Br J Ophthalmol 1978; 62: 198-209.

3 Atkin A, Bodis-Wollner I, Wolkstein M, Moss A, Podos S. Spatiotemporal contrast sensitivities in glaucoma. Invest Ophthalmol Visual Sci 1978; 17: 24.

4 Atkin A, Bodis-Wollner I, Wolkstein M, Moss A, Podos S. Abnormalities of central contrast sensitivity in glaucoma. Am J Ophthalmol 1979; 88: 205-11.

5 Atkin A, Wolkstein M, Bodis-Wollner I, Anders M, Kels B, Podos S. Interocular comparison of contrast sensitivities in glaucoma patients and suspects. Br J Ophthalmol 1980; 64: 858-62.

6 Tyler CW. Specific deficits of flicker sensitivity in glaucoma and ocular hypertension. Invest Ophthalmol Visual Sci 1981; 20: 204-12.

7 Bodis-Wollner I. Differences in low and high spatial frequency vulnerabilities in ocular and cerebral lesions. In: Maffei L, ed. Pathophysiology of the visual system. Boston: Junk, 1981: 195-204.

8 Breton ME, Spaeth GL, Wilson RP, Markoff JI. Temporal frequency loss in primary open angle glaucoma. Invest Ophthalmol Visual Sci 1982; 22: 219.

9 Towle VL, Moskowitz A, Sokol S, Schwartz B. The visual evoked potential in glaucoma and ocular hypertension: effects of check size, field size, and stimulation rate. Invest Ophthalmol Visual Sci 1983; 24: 175-83. 
10 Bobak P, Bodis-Wollner I, Harnois C, et al. Pattern electroretinograms and visual-evoked potentials in glaucoma and multiple sclerosis. Am J Ophthalmol 1983; 96: 72-83.

11 Trick GL. Anomalous PRRP spatial-temporal frequency tuning characteristics in glaucoma. Invest Ophthalmol Visual Sci 1983; 24: 104 .

12 Atkin A, Podos SM, Bodis-Wollner I. Abnormalities of the visual system in ocular hypertension and glaucoma: seeing beyond routine perimetry. In: Krieglstein GK, Leydhecker W, eds. Glaucoma update II. New York: Springer, 1983: 107-16.

SIR, Drs. Atkin et al. should not complain that their work has been inaccurately reported. In their letter they quote their report precisely and then tell us that it is neither what they meant nor what their (unpublished) data show. We would like to thank them for now confirming our result. They also stress the importance of flicker in glaucoma testing. Because we did not test patients with flicker we did not discuss their work beyond commenting on their stimulus. We now feel called upon to do so.

All clinical tests have a dual role. Those which rapidly and efficiently discriminate between affected and normal individuals come into general clinical use even when evaluated on purely pragmatic criteria. Others are normally used experimentally to tell us something about the functional or structural abnormality characterising the disorder. Such tests should be as accurate and unambiguous as possible.

We agree that tests with flickering targets can detect glaucoma better than those with static gratings. However, a maximally efficient test target should be placed in the midperiphery and need not be a grating. For example, $\mathrm{C}$. Tyler measured the full temporal modulation transfer function for a defocused spot, subtending $4^{\circ}$, centered $20^{\circ}$ above the fixation point. He reported hit rates better than $80 \%$ and few false alarms. ${ }^{1}$ The success of flickering stimuli might reflect retinal organisation in that area, the type, number, or relative density of affected to unaffected ganglion cells, cortical synaptic efficiency, or the saliency of the stimulus. It may simply be that observers, especially naive patients, respond more reliably to flickering targets, where they must repeatedly distinguish between target presence and absence, than to static ones, where they decide only once.

Atkin et al., in all 3 reports, specifically concentrated on the central visual field loss in glaucoma patients. They suggested that they had evidence of a specific loss of the $Y$ cell group, mainly because their results with flicker were the only ones which were significant. We believe that we have not misinterpreted them or misunderstood them because of the importance they gave to this interpretation.

However, their theory has little support. Only a small percentage of optic nerve fibres come from Y cells. Their diameter spectrum overlaps that of $X$ cells, so that they are unlikely to be specifically first affected by pressure damage. Although full spectra are still not available, optic fibre damage appears to be general within regions and bundles, not restricted to specific diameter groups. ${ }^{2}$ Finally, $X$ and $Y$ cells may only respond differentially to flicker well above threshold, but not at it. ${ }^{3}$ With static stimuli we could not show an interaction between contrast sensitivity loss in glaucoma and spatial frequency. Atkin et al. had to average over thresholds in structured and unstructured fields to get any significant effect. They have never shown that their effect is greater with the unstructured field, as it should be if it increases with decreasing spatial frequency. Other reasons for the loss of flicker sensitivity in disease are given above. In their letter, too, Atkin et al. present us with a second model of visual loss in glaucoma, which they had not previously advanced, but which is quite general and without implications for spatial or temporal frequency or field specific loss.

In our report ${ }^{4}$ we were concerned to develop a clinically acceptable test of the full contrast sensitivity function which could be used with any patient group. Glaucoma was discussed only as an example. We confined our discussion mainly to the many different patterns of loss which had been reported with static test stimuli.

If Drs. Atkin et al. aimed only at developing a maximally effective test of early glaucoma deficit they should have put their stimulus in the midperiphery, where the first detectable damage occurs. If they did want to say something about the underlying structural deficit in central vision in glaucoma, they have not established their point and are not misunderstood. In now stressing the importance of flicker they misrepresent the primary purpose of their work as well as our own.

Department of Clinical Ophthalmology,

VAEGAN Sydney University,

Sydney Eye Hospital,

Sir John Young Crescent,

Woolloomooloo 2011,

Australia.

Moorfields Eye Hospital,

City Rd,

London EC1V 2PD.

\section{References}

1 Tyler CW. Specific deficits of flicker sensitivity in glaucoma and ocular hypertension. Invest Ophthalmol Visual Sci 1981; 20: 204-12.

2 Quigley HA, Addicks EM, Green WR. Optic nerve damage in human glaucoma 111. Quantitative correlation of nerve fiber loss and visual field defect in glaucoma, ischemic neuropathy, papilledema and toxic neuropathy. Arch Ophthalmol 1982; 100: $135-46$.

3 Lennie P. Perceptual signs of parallel pathways. Philos Trans $R$ Soc Lond (Biol) 1980; 290: 23-37.

4 Vaegan, Halliday BL. A forced choice test improves clinical contrast sensitivity testing. Br J Ophthalmol 1982; 66: 477-91.

\section{Book reviews}

Current Management in Ophthalmology. By Douglas D. Koch, David W. Parke II, David Paton. Pp. 338. £28.50. Churchill Livingstone: Edinburgh. 1982.

This is an informative, readable, well-researched book, always entertaining, sometimes controversial, but, like any 